\title{
Horndeski scalar-tensor black hole geodesics
}

\author{
Darya Tretyakova ${ }^{1,2, \star}$, Dmitry Melkoserov ${ }^{1}$, and Timur Adyev ${ }^{1}$ \\ ${ }^{1}$ Institute for Natural Sciences, Ural Federal University, Lenin av. 51, Yekaterinburg, 620083, Russia \\ ${ }^{2}$ Sternberg Astronomical Institute, Lomonosov Moscow State University,
}

\begin{abstract}
We examine massive particles and null geodesics for the scalar-tensor black hole in the Horndeski-Galileon framework. Our analysis shows that first kind relativistic orbits, corresponding to circular and elliptic orbits, are absent for the black hole solution with the static scalar field. This is a highly pathological behavior contradicting to the black hole accretion and Solar System observations.
\end{abstract}

\section{Introduction}

Scalar-tensor gravity is a widely accepted alternative to the General Relativity for the role of gravity theory. The most general classical effective scalar-tensor theory was proposed by Horndeski [1], and the same result was aslo obtained by studying Galileons [2,3]. The Galileon model is a ghost free scalar effective field theory containing higher derivative terms that are protected by the Galileon symmetry while Horndeski was interested in a most general type of scalar-tensor theory yielding second order field equations. The resulting framework is a healthy scalar-tensor theory, which was until recently believed to be the most general scalar-tensor theory for second order field equations. A newly discovered class that goes beyond Horndeski contains no dangerous instabilities, since higher order terms cancel out due to hidden constraints [4]. However Horndeski framework remains interesting and fruitfull, working very well in cosmological applications. The action for the Horndeski/Galileon model in the non-trivial kinetic coupling sector reads

$$
S=\int d x^{4} \sqrt{-g}\left(\zeta R-\eta(\partial \phi)^{2}+\beta G^{\mu v} \partial_{\mu} \phi \partial_{\nu} \phi-2 \Lambda\right)
$$

here $G^{\mu v}$ is the Einstein tensor, $\phi$ is the scalar field, constants $\zeta>0, \eta$ and $\beta$ are model parameters. Paper [5] shows that purely kinetic coupled gravity is inconsistent with the constraint from the gravitational Cherenkov radiation for any theoretically allowed parameter $\beta$, however the action considered in [5] has $\eta=1$ and $\Lambda=0$. Introducing $\Lambda$ in the action (1) (dividing the dark energy into differentbehaving components) is a way to relax this inconsistency. The presence of a Vainshtein screening mechanism on the other hand allows the scalar field to couple to matter without mediating unacceptably large fifth forces in the solar system $[6,7]$. Henceforth it would be interesting to pursue the study of the solutions of (1) at the astrophysical scale.

Any modification of GR must be consistent with constraints astrophysical scales and at the Solar System level, which are very stringent. The purpose of this paper is thus to threat the local spherically

^e-mail: daria.tretiakova@urfu.ru 
symmetric solutions of (1) as astronomical objects and see if this picture is in agreement withe the observed one. To do so we study the test particle motion around the compact object described by the geodesic equations.

This paper is organized as follows. In section 2 we briefly summarize the properties of the black hole-like solutions for the action (1). Section 3 contains solution's parameter estimates found in the literature as well as some new ones, obtained in this paper. We proceed with analyzing geodesic equations in section 4 . Conclusions are given thereafter.

\section{Horndeski/Galileon black hole}

For the spherically-symmetric ansatz with the a mild time dependence of the scalar field

$$
\begin{aligned}
& d s^{2}=h(r) d t^{2}-\frac{d r^{2}}{f(r)}-r^{2} d \Omega^{2} \\
& \phi(r)=q t+\psi(r),
\end{aligned}
$$

black hole solutions were found in the series of papers [8]-[12]. These solutions possess very similar properties, so we choose to consider the metric from [9], the only one having peculiar terms

$$
\begin{aligned}
& f(r)=\frac{\left(1+\gamma^{2} r^{2}\right) h(r)}{\left(1+3 A r^{2}+B /\left(1+\gamma^{2} r^{2}\right)\right)}, \\
& h(r)=1-\frac{\mu}{r}+A r^{2}+B \frac{\arctan (r \gamma)}{r \gamma},
\end{aligned}
$$

where $\mu$ plays the role of the mass term. We also used the following notations ${ }^{1}$

$$
\begin{array}{rlrl}
A=\frac{\gamma^{2}}{3} \frac{2 \zeta \eta-\lambda}{2 \zeta \eta+\lambda}, & B & =\frac{\lambda^{2}}{4 \zeta^{2} \eta^{2}-\lambda^{2}}, \\
\lambda & =\zeta \eta+\beta \Lambda, & \gamma & =\sqrt{\eta / \beta} .
\end{array}
$$

By fixing all the parameters and rising $\gamma$ we approach the corresponding adS solution for $h(r)$ (which also shifts with $\gamma$ ). By lowering $\gamma$ Schwarzshild-like behavior is recovered. The solution in general interpolates in-between. However the Schwarzshild horizon radius $\mu$ is never achieved with $\gamma \rightarrow \infty$, since $r_{h}=3 / 4 \mu$ in this case and the gap remains.

\section{Parameter estimates}

In this section we briefly review the constraints on the parameters of the non-minimal derivative coupling sector of Horndeski theory, determined elsewhere. First of all, thermodynamical considerations suggest $\beta>0$ [8].

The authors of [13] considered slowly rotating neutron stars for the metric (16)-(15) by using several tabulated and realistic equations of state. The existence of a static configuration requires

$$
q^{2} \beta<4 \zeta / 3
$$

for $\beta>0$, in order to have the pressure maximum at the center of the neutron star. The model reproduces the maximal neutron mass observed and provides a viable compact objects astrophysics.

\footnotetext{
${ }^{1}$ The units are $[\gamma]=m^{-1}$.
} 
Within the constraint, it was shown that there exists neutron stars configurations with the redshift value compatible with typical measurements.

Minamitsuji [14] investigated the stability of BH solutions under massless scalar perturbations in the nonminimal derivative coupling subclass. The quasinormal modes can be computed, and no unstable modes were found. Considering the same BH solutions, Cisterna et al. [15] found that these black holes are stable under odd-parity gravitational perturbations as well. Kobayashi et al. [16] have shown that the static scalar field solution in question is stable in some range of the $\Lambda$ vs. $\zeta \gamma$ parameters.

The solution (16)-(15) possesses the Schwarzschild - anti de Sitter structure if we suggest that $\Lambda$ does not exceed the observed value $\Lambda \approx 10^{-52} \mathrm{~m}^{-2}$. For the $\Lambda$ CDM expansion history, there essentially always exists a family of models which interpolate between the cosmological constant as the only source of acceleration and models which are fully dynamical, so the conjecture above makes sense. Given this value of $\Lambda$ we can further neglect it with respect to the other terms and set $\Lambda \approx 0$. Then

$$
\lambda \approx \zeta \eta, \quad A \approx \frac{\gamma^{2}}{9}, \quad B \approx \frac{1}{3} .
$$

With this simplifications we have now only one additional parameter $\gamma$ left for the fine tuning. The correction term

$$
\Delta(r)=B \frac{\arctan (r \gamma)}{r \gamma}
$$

now gives $\Delta \rightarrow B$ near $r=0$ and $\Delta \rightarrow 0$ when $r \rightarrow \infty$.

We can also consider weak-field observations. One of the well-studied gravitational effects is the frequency shift for the satellite on the Earth orbit

$$
\frac{\delta v}{v}=1-\sqrt{\frac{h(R+h)}{h(R)}} \approx \frac{V(R+h)-V(R)}{c^{2}},
$$

up to the first order in the weak field approximation with $h$ being the satellite orbit height and $R$ - the Earth surface radius, $V$ - the corresponding gravitational potential. For the metric (4)-(5) there should be an additional shift, related to the deviation of $h(r)$ from the Schwarzshild solution

$$
\begin{aligned}
& 2 \frac{\delta v}{v} \approx \delta_{S c h w}+\delta_{1}+\delta_{2}= \\
& \quad\left(\frac{\mu}{R}-\frac{\mu}{R+h}\right)+A\left((R+h)^{2}-R^{2}\right)+(\Delta(R+h)-\Delta(R)) .
\end{aligned}
$$

Modern frequency measurements are in agreement with GR, hence we can make bounds using the frequency measurement accuracy $10^{-14}$ achieved in the GP-A redshift experiment ${ }^{2}$ [17]. Numerical estimates show that $\delta_{1}+\delta_{2}$ does not exceed the accuracy of the relative frequency measurement when

$$
\gamma<10^{-14}
$$

This estimate allows us to set $A \approx 0$ (and hence $\gamma^{2} \approx 0$ ) when considering accretion and null geodesics for the Sgr A ${ }^{3}$ black hole ore any smaller one since the conditions $A r^{2}<<\mu / r$ and $A r^{2}<<\Delta$ are well

\footnotetext{
${ }^{2} \mathrm{~h}=15 \times 10^{3} \mathrm{~km}, M_{\oplus}=5,972 \times 10^{24} \mathrm{~kg}, R_{\oplus}=6371 \mathrm{~km}$, $G_{0}=6.67384 \times 10^{-11} \mathrm{~m}^{3} \mathrm{~kg}^{-1} \mathrm{c}-2$

${ }^{3} M_{S g r A}=4 \times 10^{6} M_{\odot}$
} 
satisfied within the corresponding $100 r_{S c h w}$ distance. All the above results in the metric

$$
\begin{aligned}
h(r) & =1-\frac{\mu}{r}+\frac{1}{3} \frac{\arctan (r \gamma)}{r \gamma}, \\
f(r) & =\frac{3}{4} h(r) .
\end{aligned}
$$

\section{Geodesic equations}

Geodesic equation for the radial coordinate reads

$$
\begin{aligned}
& \left(\frac{d r}{d \varphi}\right)^{2}=f(r) P(r), \\
& P(r)=\left[\frac{E^{2}-j h(r)}{h(r)}\right] \frac{r^{4}}{L^{2}}-r^{2},
\end{aligned}
$$

where $j=0$ for massless particles and $j=1$ for the massive ones. We will further use the inverse radius $u=r^{-1}$. Working with geodesics we are beyond the black hole horizon and hence $h(u) \neq 0$ (and hence $f(u) \neq 0$ ). Therefore we can write (18) for the circular orbits as

$$
\begin{aligned}
P(u) & =0 \\
P^{\prime}(u) & =0 .
\end{aligned}
$$

\subsection{Null geodesics}

An observational parameter, which will be important in the near future, is the photon sphere radius of the black hole, which relates directly to it's shadow size. Due to the smallness of $\gamma$ the following expansion will be valid not too far from the black hole:

$$
\begin{aligned}
& \arctan (r \gamma) \approx r \gamma-\frac{(r \gamma)^{3}}{3}, \\
& \Delta(r) \approx \frac{1}{3}-A r^{2} \\
& h(r) \approx \frac{4}{3}-\frac{\mu}{r} .
\end{aligned}
$$

Writing the null geodesic equation in terms of the inverse radius $u=r^{-1}$ we thus obtain

$$
\begin{aligned}
& P(u)=\frac{E^{2}}{h(u)} \frac{u^{-4}}{L^{2}}-u^{-2} \\
& \left(\frac{d u}{d \varphi}\right)^{2}=\frac{3}{4} \frac{E^{2}}{L^{2}}-u^{2}+\frac{3}{4} \mu u^{3}=R(u)
\end{aligned}
$$

We are seeking for the last unstable photon orbit, so we are interested in the critical case when two positive real roots of $R(u)$ coincide, which gives in our case

$$
u_{1}=u_{2}=\frac{8}{9 \mu}, \quad u_{3}=-\frac{4}{9 \mu} .
$$


The solution of the geodesic equation takes then the following form:

$$
u=-\frac{4}{9 \mu}+\frac{4}{3 \mu}\left[\tanh \left(\frac{\varphi-\varphi_{0}}{2}\right)\right]^{2} .
$$

Considering the limit $\varphi \rightarrow \infty$ we can find the radius of photon sphere as

$$
r_{s}=\frac{9 \mu}{8}
$$

whereas $r_{s}=3 \mu / 2$ for the Schwarzshild metric. The observational characteristics of the metric in question differ sufficiently and require therefore further exploration, so we now proceed with timelike geodesics.

\subsection{Massive particles}

For the bound orbits we have $E^{2}<1$. Substituting (15) into (20)-(21) we obtain

$$
\begin{gathered}
\mu u^{3}-u^{2}+\frac{j \mu}{L^{2}} u-\frac{j-E^{2}}{L^{2}}-\Delta\left(u^{2}+\frac{j}{L^{2}}\right)=0, \\
3 \mu u^{2}-2 u+\frac{j \mu}{L^{2}}-\Delta^{\prime}\left(u^{2}+\frac{j}{L^{2}}\right)-2 u \Delta=0,
\end{gathered}
$$

where the prime denotes the derivative with respect to the radial coordinate. One can simplify $\Delta^{\prime}$ if we recall that $\gamma^{2} / u^{2} \equiv \gamma^{2} r^{2} \sim A r^{2}$, and neglect this term as we did before. Now extract $\Delta$ from (31) and substitute into (32):

$$
\begin{aligned}
F(u) & =(1-3 \varepsilon) \mu u^{3}-\left(1-\frac{5}{3} \varepsilon\right) u^{2}+(1-\varepsilon) \frac{\mu}{L^{2}} u-\frac{(1+\varepsilon / 3)-E^{2}}{L^{2}}, \\
\varepsilon & =1-\frac{2}{3+\frac{1}{u^{2} L^{2}}},
\end{aligned}
$$

where $F(u)=0$. Note that $\varepsilon(u, L)$ is bound as $1 / 3<\varepsilon<1$ while $u$ is running between 0 and $\infty$ : This forces the coefficient before $u^{3}$ in $F(u)$ to be negative and determines the behavior of the expression. We can also rewrite $F(u)$ as

$$
F(u)=\frac{1}{3} \frac{-4 L^{4} u^{4}+9 E^{2} L^{2} u^{2}-8 L^{2} u^{2}+3 E^{2}-4}{\left(3 L^{2} u^{2}+1\right) L^{2}}
$$

which is a direct consequence of $B=1 / 3$ since higher orders in $u$ cancel out in the equation. This results in the following solution for the circular geodesics:

$$
u= \pm \frac{1}{4 L} \sqrt{18 E^{2}-16 \pm 2 \sqrt{81 E^{4}-96 E^{2}}}
$$

We see that for $E^{2}<1$ there are no real positive roots, so circular orbits for massive particles are excluded. This may seem an artifact of the approximation, however one can verify numerically for the general metric (5), that unlike the Schwarzshild case conditions (20) and (21) can never be satisfied simultaneously.

Circular orbits represent a narrow subclass of relativistic bound orbits. However, as they represent a subclass of the elliptic orbits, the latter should be excluded as well ( the analysis does not involve the eccentricity at all) and so all the first class orbits, twisted between two radii are eliminated. This conclusion can be also obtained by analysing the general behavior of $\mathrm{P}(\mathrm{u})$. This leaves us only with the trajectories, ending up in the black hole singularity $(\varepsilon)$. 


\section{Discussion and conclusions}

Departing from GR we are left aside of the Birkhoff's theorem. In this situation we are forced to choose between multiple spherically symmetric vacua and therefore some hints on how to determine the realistic solution would come in handy. A configuration used by the authors of [9] is rather interesting. By introducing a mild linear dependence in the time coordinate for the scalar field one evades the scalar field being singular for it's derivative on the horizon [18] and makes the field equations to bifurcate the no-hair theorem at the same time. Despite this dependence the shift symmetry is keeping the field equations time-independent and consistent with the static ansatz. This permits asymptotically flat (or de-Sitter) solutions and crucially gives regular scalar tensor black holes.

The metric (4)-(5) is static, but has $q=0$ for the the scalar field which is not regular at the horizon. Our analysis of this metric revealed the absence of stable orbits which do not end up in the singularity. This is a highly pathological behavior contradicting to the black hole accretion observations [19, 20]. Furthermore, the Schwarzschild solution in GR also describes the exterior of any spherically symmetric body in the weak field limit (hence the Solar System), and so must do it's analog in extended gravity theory. However, with in-falling orbits only, the metric (4)-(5) fails to reproduce the solar system motion. This is the situation that to our knowledge did not appear before in extended gravity solutions: usually we can always suppress the deviations from the GR by the fine tuning. Another interesting example, demonstrating that studying local spherically symmetric solutions can provide useful information on cosmological models is given in [21]. The paper implements some PPN-based bounds on the model parameters due to the black hole-like metric for the framework, initially constructed for cosmological purposes. The parameter region left does not satisfy the purposes of the model, rendering it far less attractive. Hence geodesic analysis remains a useful tool to explore extended gravity solutions, even though the observations of the test body motion in the vicinity ob black holes are not sensitive enough to distinguish extended gravity from the GR. Black hole geodesics consideration can give us the direction towards astrophysically viable black holes in Horndeski-Galileon framework.

\section{Acknowledgements}

This work was supported by Russian Foundation for Basic Research via grant RFBR №16-02-00682.

\section{References}

[1] G.W. Horndeski, Int. J. Theor. Phys. 10, 363 (1974)

[2] C. Deffayet, S. Deser, G. Esposito-Farese, Phys. Rev. D80, 064015 (2009), 0906. 1967

[3] C. Deffayet, G. Esposito-Farese, A. Vikman, Phys. Rev. D79, 084003 (2009), 0901.1314

[4] C. Lin, S. Mukohyama, R. Namba, R. Saitou, JCAP 1410, 071 (2014), 1408.0670

[5] R. Kimura, K. Yamamoto, JCAP 1207, 050 (2012), 1112 . 4284

[6] R. Kase, S. Tsujikawa, JCAP 1308, 054 (2013), 1306.6401

[7] E. Babichev, C. Deffayet, G. Esposito-Farese, Phys. Rev. Lett. 107, 251102 (2011), 1107.1569

[8] M. Rinaldi, Phys. Rev. D86, 084048 (2012), 1208.0103

[9] E. Babichev, C. Charmousis, JHEP 1408, 106 (2014), 1312 . 3204

[10] C. Charmousis, D. Iosifidis, J. Phys. Conf. Ser. 600, 012003 (2015), 1501.05167

[11] E. Babichev, C. Charmousis, M. Hassaine, JCAP 1505, 031 (2015), 1503.02545

[12] M. Minamitsuji, Phys. Rev. D89, 064017 (2014), 1312 . 3759 
[13] A. Cisterna, T. Delsate, L. Ducobu, M. Rinaldi, Phys. Rev. D93, 084046 (2016), 1602 . 06939

[14] M. Minamitsuji, Gen. Rel. Grav. 46, 1785 (2014), 1407.4901

[15] A. Cisterna, M. Cruz, T. Delsate, J. Saavedra, Phys. Rev. D92, 104018 (2015), 1508.06413

[16] T. Kobayashi, H. Motohashi, T. Suyama, Phys. Rev. D89, 084042 (2014), 1402 . 6740

[17] R.F.C. Vessot, M.W. Levine, E.M. Mattison, E.L. Blomberg, T.E. Hoffman, G.U. Nystrom, B.F. Farrel, R. Decher, P.B. Eby, C.R. Baugher et al., Phys. Rev. Lett. 45, 2081 (1980)

[18] C. Charmousis, T. Kolyvaris, E. Papantonopoulos, M. Tsoukalas, JHEP 07, 085 (2014), 1404.1024

[19] J.A. Muñoz, E. Mediavilla, C.S. Kochanek, E.E. Falco, A.M. Mosquera, The Astrophysical Journal 742, 67 (2011), 1107. 5932

[20] R. Fender, T. Belloni, Science 337, 540 (2012), 1208. 1138

[21] D.A. Tretyakova, B.N. Latosh, S.O. Alexeyev, Class. Quant. Grav. 32, 185002 (2015), 1504.06723 